www.jmscr.igmpublication.org

Impact Factor 5.84

Index Copernicus Value: 71.58

ISSN (e)-2347-176x ISSN (p) 2455-0450

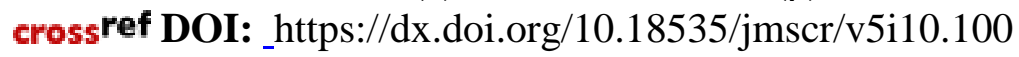

Journal Of Medical Science And Clinical Research

\title{
Prognostication of NLR and PLR in Oral Squamous Cell Carcinoma
}

\author{
Authors \\ Dr Ananthaneni Anuradha, Dr Penumala Vignatha, Dr G. Vijay Srinivas, \\ Dr Bagalad S Bhavana, Dr Md. Asif Kiresur, Dr Puneeth HK \\ St. Joseph Dental College, Duggirala, Eluru, Andhra Pradesh
}

\section{Abstract}

Background: Inflammatory status of host plays an important role in assessing the prognosis in cancer patients. Neutrophil lymphocyte ratio (NLR) and platelet neutrophil ratio (PLR) reflect the status of systematic inflammation. These novel markers have emerged as valuable and reliable prognostic and mortality evaluators in cancer patients. So we aimed to determine the prognostic significance of these inflammatory indices in oral squamous cell carcinoma patients.

Objective: The purpose of this study is to determine the prognostic value of PLR and NLR in patients with resected oral squamous cell carcinoma who had undergone concurrent chemotherapy and radiotherapy (CTRT).

Method: We performed a retrospective analysis of 20 oral carcinoma patients who underwent concurrent CTRT following resection. The prognostic value of the laboratory parameters were evaluated by ' $t$ ' test and univariate analysis.

Results: The statistical analysis revealed that there was an increase in NLR and PLR post treatment but the increase was statistically significant only in PLR. Univariate analysis was done pertaining to parameters such as age, gender, NLR, PLR, PFS (progression free survival) and OS (overall survival) to evaluate the association between these factors and PFS and OS.

Conclusion: This study provided evidence for the association between NLR, PLR to outcome in oral squamous cell carcinoma. Preoperative NLR, PLR may represent a simple and cost-effective method for predicting the prognosis.

Keywords: Neutrophil Lymphocyte Ratio, Platelet Neutrophil Ratio, Progression Free Survival, Overall Survival.

\section{Introducción}

Oral cancer accounts for $2 \%-4 \%$ of all cancer cases. In some regions, the prevalence of oral cancer is higher, reaching the $45 \%$ of all cancers in India ${ }^{[1,2]}$. It is estimated that more of $90 \%$ of all oral neoplasms are OSCC. ${ }^{[3]}$ Patient prognosis depends on multiple variables including patientspecific characteristics (e.g. performance status, age, and race), tumour biology (e.g. tumour size, nodal status, histologic grade), and response to systemic therapy. Inflammation is also likely to be an important marker of outcome in cancer. In cancer, clinical outcome may be influenced not only by the histopathological characteristics of the tumour itself, but also by the host response, including the inflammatory response. ${ }^{[3]}$ Cellular mediated inflammatory response, lymphocytes, neutrophils, and monocytes are increasingly being 
recognised as having an important role in tumorigenesis and carcinogenesis. Therefore, predictive biomarkers reflecting the response of this disease to these agents may help to guide management. Haematological indices, such as neutrophil-to-lymphocyte ratio (NLR), platelet-tolymphocyte ratio (PLR) have been to assess inflammatory response. ${ }^{[4]}$

Therefore, we conducted the present study to evaluate the association of pretreatment levels of NLR or PLR with the clinical outcome of advanced oral Squamous cellcarcinoma patients, who were treated with cysplatin chemotherapy.

\section{Methods}

\section{Study population}

20 patients were considered in this study. All the patients had histologically confirmed oral Squamous cell carcinoma. All the patients were aged between 20 and 70 years. These patients were treated with concurrent chemotherapy and radiotherapy. Cysplatin was the drug of choice.

\section{Follow-up evaluation and assessment of response}

Before each treatment courses, a physical examination, routine hematology, biochemistry, and chest X-ray were carried out. Computed tomography scans to define the extent of the disease, and the responses were carried out after two cycles of chemotherapy.

\section{Blood Sample Analysis}

Venous blood samples were taken from patients admitted to the oncology outpatient clinic for palliative chemotherapy, and were collected in ethylene diamine tetraacetic acid (EDTA)containing tubes. WBC differential counts were analyzed by

The NLR was calculated from the differential count by dividing the neutrophil measurement by the lymphocyte measurement. An NLR 3 or greater was considered as elevated. PLR was evaluated as platelet count divided by lymphocyte count. The calculated values were divided into two categories as $<160$ or $\geq 160$. Both NLR and PLR were recorded at baseline and where available after 1 cycle of systemic therapy.

\section{Statistical analysis}

The associations between NLR or PLR and the clinicopathologic parameters (sex, age, gender) was considered. The progression free survival (PFS) and overall survival (OS) were calculated from the date of initiation of therapy to the date of disease progression and death, respectively. Patients who were alive at the last follow-up were censored at that time. Patients, who were taken off from the study or who died before progressions were censored at the time when they were taken off from the study. The association of each marker with survival was analyzed using paired t test and univariate analysis.

\section{Results}

\section{Patient characteristics}

From January 2014 to December 2016, a total of 20 patients enrolled in the present study. Demographic details about the patients included in the present study are shown in Table 1 . Overall, there were $13(65 \%)$ male and $7(35 \%)$ female patients. All of the patients underwent operation and received an adjuvant chemotherapy with cysplatin. None of the patients showed clinical signs of sepsis or other inflammatory illnesses at the time of commencement of systemic therapy.

\section{Prognostic variables according to NLR and PLR}

Correlations between the NLR and clinicopatholotic parameters are shown in Table. The NLR was grouped with respect to 2 different cutoff points $(<3$ or $\geq 3)$. Eleven patients $(55 \%)$ were detected with NLR of less than 3 , while there were 9 patients $(45 \%)$ whose NLR was greater than or equal to 3. The PLR was grouped with respect to 2 different cutoff points $(<160$ or $\geq 160$ ). There were 8 patients with PLR $<160$ $(40 \%)$ and 12 patients with PLR $<160$. 


\section{Association of NLR or PLR with survival}

The univariate analysis was performed and the results for the predictors of survival are listed in Table 1. Univariate analysis revealed that old age was a predictor of worse PFS and overall survival. Patients with NLR $\geq 3$ showed shorter OS than patients with NLR of less than 3. Patients were categorized into 3 groups according to the changes in NLR after first cycle of chemotherapy.

1) NLR $<3$ at baseline and after 1 cycle of chemotherapy

2) NLR $<3$ at baseline and $\geq 3$ after 1 cycle of chemotherapy

3) NLR $\geq 3$ at baseline and after 1 cycle of chemotherapy

Patients with lower NLR before second cycle of chemotherapy had an improved PFS when compared with patients with higher NLR. Patients with persistently elevated NLR showed lower PFS and OS. There was an inclination towards a shorter survival rates when the PLR was $\geq 160$ compared with less than 160. Patients were categorized into 3 groups according to the changes in PLR after first cycle of chemotherapy.

1) PLR < 160 at baseline and after 1 cycle of chemotherapy

2) PLR $<160$ at baseline and $\geq 160$ after 1 cycle of chemotherapy

3) PLR $\geq 160$ at baseline and after 1 cycle of chemotherapy

Patients with lower PLR before second cycle of chemotherapy had an improved PFS when compared with patients with higher PLR. Patients with persistently elevated PLR showed lower PFS and OS. Patients with PLR equal or higher than 160 before and after 1 cycle of chemotherapy showed low survival rates.
Table 1: prognostic factors in univariate analysis. NLR- neutrophil lymphocyte ratio; PLR- platelet lymphocyte ratio; OS - overall survival; PFS progression free survival.

\begin{tabular}{|l|l|l|l|l|}
\hline \multicolumn{5}{|c|}{ PROGNOSTIC FACTORS IN UNIVARIATE ANALYSIS } \\
\hline S.NO & PFS (IN MONTHS) & PFS & OS(IN MONTHS) & OS \\
\hline & MEAN & P VALUE & MEAN & P VALUE \\
\hline 1.AGE & & & & \\
\hline$<60$ YEARS & 3.16 & 0.78 & 4.16 & 0.99 \\
\hline$>60$ YEARS & 2.6 & 0.61 & 3.60 & 0.34 \\
\hline 2.GENDER & & & & \\
\hline MALE & 2.93 & 0.26 & 3.93 & 0.83 \\
\hline FEMALE & 3.70 & 1.00 & 5.00 & 1.00 \\
\hline $3 . N L R$ & & & & \\
\hline NLR<3 & 3.9 & 1 & 6.4 & 1 \\
\hline NLR >3 & 2 & 0.00 & 3 & 0.00 \\
\hline $4 . P L R$ & & & & \\
\hline PLR<160 & 3.23 & 0.98 & 4.23 & 0.99 \\
\hline PLR>160 & 1 & 0.70 & 2 & 0.72 \\
\hline $\begin{array}{l}\text { CHANGE IN NLR } \\
\text { AFTER CHEMO }\end{array}$ & & & & \\
\hline NLR $<3-<3$ & 5.4 & & & \\
\hline NLR <3->3 & 2.3 & 1 & 6.4 & 1 \\
\hline NLR >3->3 & 2 & 0.00 & 3.33 & 0.023 \\
\hline $\begin{array}{l}\text { CHANGE IN PLR } \\
\text { AFTER CHEMO }\end{array}$ & & 0.00 & 3 & 0.00 \\
\hline PLR <160 -<160 & 3.83 & & & 0.00 \\
\hline PLR <160->160 & 1.80 & 0.99 & 4.833 & \\
\hline PLR $>160->160$ & 1 & 0.00 & 2.80 & \\
\hline
\end{tabular}

\section{Discussion}

Inflammation is a critical component of tumour progression. It now a known fact that the tumour microenvironment, which is studded with inflammatory cells, is an essential participant in the neoplastic process, fostering proliferation, survival and migration. Tumour cells produce various cytokines and chemokines that attract leukocytes. The inflammatory component of a developing neoplasm include neutrophils, dendritic cells, macrophages, eosinophils and mast cells, as well as lymphocytes - all of which are capable of producing cytokines, cytotoxic mediators including reactive oxygen species, serine and cysteine proteases, MMPs and membrane-perforating agents, and soluble mediators of cell killing, such as TNF- $\alpha$, interleukins and interferons (IFNs). ${ }^{[5]}$

Neutrophils also influence the tumor microenvironment, including stromal cells, the vasculature, and other immune cells. Angiogenesis is required to create the tumor vasculature, which particularly in larger tumors is 
critical to provide the proliferating cancer cells with oxygen and nutrients. This process can be promoted by MMP-9 in tumors. MMP9 cleaves the heparin-binding domain of VEGF, thereby releasing VEGF from the ECM and increasing angiogenesis. Neutrophils are a major source of MMP-9 and produce it. unlike other cells, in the absence of its inhibitor TIMP-1, resulting in high levels of active MMP9.

MMP-9 was shown to also collaborate with MMP-2 in promoting the in vivo invasive and angiogenic phenotype of cancer cells. ${ }^{[6]}$

Tumor progression is characterized by a complex network of relationships among different cell types that collectively exploit a metabolic reprograming and mutually influence their functionality and, in particular, $\mathrm{T}$ cell functions. Because of the Warburg effect and high glucose consumption by cancer cells, tumor microenvironment shows reduced extracellular concentration of glucose. As a result of glucose deprivation, tumor-infiltrating $\mathrm{T}$ cells decrease aerobic glycolysis and generation of the phosphoenolpyruvate (PEP) metabolite involved in $\mathrm{T}$ cell receptor dependent activation of $\mathrm{Ca}^{2+}$, thus losing their antitumoral effector function. Acidification of tumor microenvironment dramatically impairs cytotoxic $\mathrm{T}$ cell proliferation and function. Accelerated fermentation, generating high level of lactate, constitutes a marker for metastases and correlates with poor prognosis. Also hypoxia represents a hindrance to $\mathrm{T}$ cell antitumor responses. HIF- $1 \alpha$ has been shown to upregulate the expression of PD-1 ligand on cancer cells, thus inhibiting $\mathrm{T}$ cell-mediated cytotoxicity. Amino acid metabolism also represents a crucial process in tumor progression. In particular, L-arginine and tryptophan catabolism have been demonstrated to be dysregulated in cancers. ${ }^{\text {[7] }}$

Platelet activity may be involved in tumor metastasis. The tumor cells, after detachment from the primary site, adhere to vascular endothelium at distant sites and proliferate. Platelets form aggregates with tumor cells in circulation, facilitating their adhesion to the vascular endothelium. Formation of platelet-tumor cell aggregates and their sequestration in various endorgans may result in thrombocytopenia. Certain tumor cell lines directly stimulate platelet activity, some by releasing platelet-aggregating material, a urea-extractable membrane component, others by release of cathepsin, and still others by undefined mechanisms. The direct effect of platelets on tumor cells may be of pathogenic significance. For example, platelet-derived factors stimulate growth of some tumors, whereas others increase vascular permeability and thus facilitate migration of tumor cells across the vessel wall. ${ }^{[8]}$

Since the inflammatory components play a crucial role in cancer we tried to study the association between two indices NLR and PLR to survival rates and found that these act as good prognostic factors.

\section{Conclusion}

Thus we can conclude that pre and post treatment routine hematological parameters such as NLR and PLR can be used as prognostic indicators in patients with oral Squamous cell carcinoma. These indices are potential predictor of the pathologic response to chemotherapy. The low cost and easy accessibility of complete blood picture are other features that enhances the use of these indices.

\section{References}

1. Williams HK. Molecular pathogenesis of oral squamous carcinoma.Mol Pathol 2000; 53: 165-72.

2. Siddiqui IA, Farooq MU, Siddiqui RA, Rafi SMT. Role of toluidine blue in early detection of oral cancer. Pak J Med Sci 2006;22:184-7.

3. Choi S, Myers JN. Molecular pathogenesis of oral squamous cell carcinoma: implications for therapy. J Dent Res 2008; 87: 14-32.

4. Sara Socorro Faria et al. The neutrophil-tolymphocyte ratio: a narrative review. 
ecancer 2016, 10:702 DOI: 10.3332/ ecancer.2016.702.

5. Lisa M. Coussens et al. Inflammation and cancer. Nature. 2002 December 19; 420(6917): 860-867.

6. Louise W. Treffers. Neutrophils in cancer. Immunological reviews.2016;273(1), 312328.

7. Barbara Molon, Bianca Calì and Antonella Viola. T Cells and Cancer: How Metabolism Shapes immunity. Frontiers in Immunology;2016;7(20):1-7.

8. P Mehta. Potential role of platelets in the pathogenesis of tumor metastasis. 